\title{
Modulation of Hydrophilic/Hydrophobic Character of Porous Environments in Metal-Organic Frameworks via Direct Polymer Capping Probed by NMR Diffusion Measurements
}

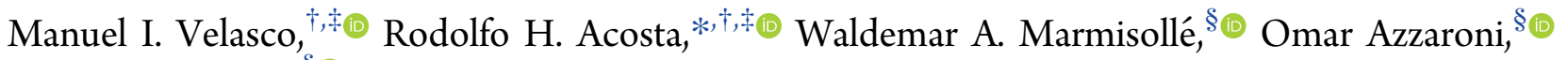 \\ and Matías Rafti* ${ }^{\S}{ }_{(0)}$ \\ ${ }^{\dagger}$ Facultad de Matemática, Astronomía, Física y Computación, Universidad Nacional de Córdoba, 5016 Córdoba, Argentina \\ ‡CONICET Instituto de Física Enrique Gaviola (IFEG), 5016 Córdoba, Argentina \\ ${ }^{\S}$ Instituto Investigaciones Fisicoquímicas Teóricas y Aplicadas (INIFTA), Universidad Nacional de La Plata-CONICET, CC 16, \\ Suc. 4, 1900 La Plata, Argentina
}

Supporting Information

ABSTRACT: The ability to create extended porous networks, such as those composed of metal-organic frameworks (MOFs), with tailored hydrophilic/hydrophobic character is crucial for adapting such widely used supports to different applications. To achieve this goal, direct polymer inclusion has proven to be a suitable strategy, and functional composite materials with multiple additional properties have been obtained in such a way. We have explored, by means of nuclear magnetic resonance diffusion experiments, the effect of polymer capping using a conducting polymer (polyaminobencylamine-PABA), which results in the positioning of $-\mathrm{NH}_{2}$ moieties on the otherwise eminently hydrophobic surface of Zn-based ZIF-8 MOF nanocrystals. Our results demonstrate that increasingly higher amounts of PABA can confer, aside from conductivity, an increased hydrophilic character to the porous network, while also allowing for the identification of different environments available for water molecule diffusion.

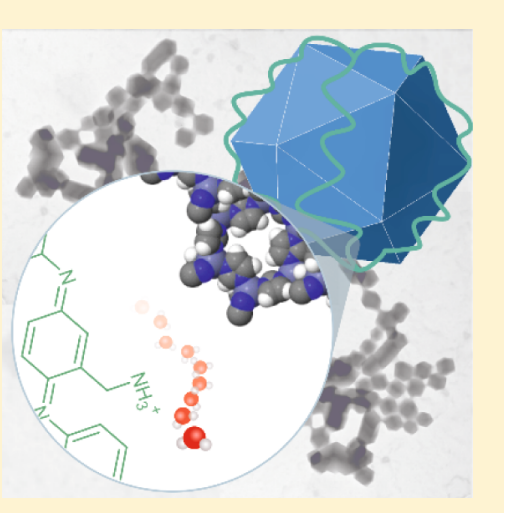

\section{INTRODUCTION}

Traditional porous materials, such as carbon-based solids or zeolites, have attracted a great deal of attention in the last decades because of their extensive use in the construction of complex architectures for many important applications ranging from catalysis, sensing, or separation technologies, to drug delivery, energy storage, and conversion devices. In this context, an exciting and relatively new class of porous materials has emerged, which can be best described as porous coordination polymers formed by an ordered tridimensional framework of metal ions (or metal-containing clusters) coordinated by multidentate organic linkers. These materials are widely known as metal-organic frameworks (MOFs). ${ }^{1-3}$ Triggered by their versatility with regard to morphologies, ${ }^{4}$ chemical composition, ${ }^{5}$ surface chemistry, ${ }^{6}$ and possible integration into diverse platforms and supramolecular coordination complexes, ${ }^{7,8}$ the number of published studies dedicated to the exploration of MOF-based applications is continuously increasing. To name a few interesting energyrelated examples which take advantage of the above-described features, it was recently shown that MOFs are capable of enzyme-like complexity when conveniently modified; ${ }^{9}$ they were also used in photoelectric energy conversion applications, ${ }^{10,11}$ integrated into $\mathrm{Li}-\mathrm{O}_{2}$ cell cathode materials, ${ }^{12}$ or used for $\mathrm{CO}_{2}$ capture and photocatalytic conversion. ${ }^{13,14}$
Many of the mentioned exciting applications require the use of MOFs which would remain stable in aqueous phase or water-containing environments, ${ }^{15,16}$ and although there are several examples of MOFs which comply with this condition, surface modification strategies have been proved effective to confer additional stability, thus widening the available options. ${ }^{17-20}$ Soft polymeric electrodes are cost-effective and very versatile materials used for many applications, ${ }^{21,22}$ such as the promotion of the oxygen reduction reaction (ORR) at neutral $\mathrm{pH}$ in aqueous environments. Synergy between an electroactive component (polyaminobencylamine-PABA, a conducting polymer (CP) featuring $-\mathrm{NH}_{2}$ moieties) and a high surface area material capable of enhancing gas adsorption (e.g., ZIF-8 MOF, constituted by tetrahedrally coordinated $\mathrm{Zn}^{2+}$ ions with 2-methylimidazolate $)^{19,23,24}$ was explored and proved to be successful to create a soft composite electroactive surface, featuring both meso- and microporosity. ${ }^{25,26}$ The observed enhancement of the ORR can be understood in terms of an interplay between the eminently hydrophobic (and capable of oxygen adsorption) microporosity present in ZIF-8 crystals (the reported Brunauer-Emmett-Teller surface areas range between 1500 and $1700 \mathrm{~m}^{2} \mathrm{~g}^{-1}$, with micropores of $\sim 0.3$ $\mathrm{nm}$ window, and $\sim 1.1 \mathrm{~nm}$ diameter size $)^{24}$ and the PABA-

Received: July 17, 2019

Published: August 1, 2019 
coated interparticle mesoporosity. ${ }^{27}$ It was hypothesized that the hydrophilicity endowed by PABA allows the (otherwise hindered) diffusion of ionic species intervening in the ORR, in line with what was reported for similar systems of polymercapped MOF films. ${ }^{28}$

A crucial parameter that determines the performance of any porous material for a given application is the transport rate of different species through the framework. Although nuclear magnetic resonance (NMR)-related techniques have been extensively used for MOFs' structural and functional characterization; ${ }^{29-31}$ it was only recently that NMR diffusion measurements were explored as a tool for acquiring information regarding pore dimension and its chemical nature, network tortuosity, connectivity, and (specially for mesoporous environments) even molecular density of the adsorbed phase. $^{32-34}$ Additionally, in partially saturated mesopores, diffusion measurements of the liquid phase render information on the molecular exchange between the gas and liquid phases. $^{35}$ Although a few fundamental studies on the adsorption/diffusion dynamics of simple molecules on $\mathrm{MOFs}^{30,36}$ or even the effect of subtle framework modifications on the adsorbed-phase translational and rotational degrees of freedom have been recently published, ${ }^{37}$ the challenging problem of understanding and controlling the behavior of adsorbed water on MOFs has only been partially addressed to the best of our knowledge. ${ }^{38,39}$

Having in mind the above discussion and the importance of the described micro/mesoporous films as electrochemical platforms, we have explored the behavior of adsorbed water in polymer-MOF composites constituted by PABA/ZIF-8. Our main goal was to characterize the effect of polymer inclusion on the hydrophobic/hydrophilic character of different porous environments present, using as benchmark a wateradsorbing Al-trimesate-based MOF, MIL-96. ${ }^{38,40,41}$ Samples were exposed to water-saturated atmosphere to populate the different porous environments. The determination of the diffusion coefficients of water was carried out using a singlesided NMR spectrometer. ${ }^{42}$ For each sample, a different distribution of diffusion coefficients was obtained, from which the relative amount of water molecules with different mobilities can be determined. Our results reveal that different environments for water molecules are present in the prepared polymer-MOF samples, as it could be hypothesized from the interplay between the well-known ZIF-8 hydrophobic character (that could be traced back to $-\mathrm{CH}_{3}$ moieties present on 2-methylimidazolate linkers) and PABA. As the PABA content in the composite is augmented, an overall hydrophilicity increase can be observed together with the emergence of a new available mesoscopic-macroscopic environment, compatible with the polymer-coated interparticle space.

\section{EXPERIMENTAL SECTION}

Methanol, ammonium persulfate (APS), 3-aminobenzylamine (ABA), 2-methyl-imidazole, aluminum nitrate $\left(\mathrm{Al}\left(\mathrm{NO}_{3}\right)_{3}\right.$. $\left.9 \mathrm{H}_{2} \mathrm{O}\right)$, and zinc nitrate hexahydrate $\left(\mathrm{Zn}\left(\mathrm{NO}_{3}\right)_{2} \cdot 6 \mathrm{H}_{2} \mathrm{O}\right)$ were purchased from Sigma-Aldrich. 1,3,5-Benzenetricarboxylic acid $95 \%\left[\mathrm{C}_{6} \mathrm{H}_{3}\left(\mathrm{CO}_{2} \mathrm{H}\right)_{3}\right.$ or benzene-1,3,5-tricarboxylic acid $\left(\mathrm{H}_{3}\right.$ btc $\left.)\right]$ was purchased from Aldrich. Aluminum MIL-96 MOF was synthesized following the procedure published by Loiseau et al., ${ }^{40}$ that is, under a hydrothermal autogenous pressure using a Teflon-lined steel Parr autoclave of a mixture of aluminum nitrate and $\mathrm{H}_{3}$ btc in water (molar ratio 1:0,14:80; $24 \mathrm{~h}$ at $210{ }^{\circ} \mathrm{C}$ ). The powder product obtained was filtered and washed with fresh methanol and dried in vacuum at 120 ${ }^{\circ} \mathrm{C}$. Colloidal synthesis of ZIF-8 MOF was carried out according to already published procedures. Briefly, a direct room-temperature mixture of $25 \mathrm{mM}$ methanolic $\mathrm{Zn}\left(\mathrm{NO}_{3}\right)_{2}$. $6 \mathrm{H}_{2} \mathrm{O}$ and $50 \mathrm{mM}$ 2-methylimidazole solutions (typically, 10 $\mathrm{mL}$ of each for a final stoichiometric 1:2 molar ratio) was prepared. $^{25}$ Following subsequent centrifugation and redispersion steps with a fresh solvent, $200 \mathrm{~nm}$ nanocrystals of uniform size distribution can be obtained (see Supporting Information).

The synthesis of PABA was carried out according to procedures published elsewhere. ${ }^{43}$ Polymerization was achieved by chemical oxidation by APS. Briefly, a $50 \mathrm{mM}$ $\mathrm{ABA}$ solution was prepared by dissolving the solid in water, obtaining a $\mathrm{pH}$ of $9-10$. After 15 min of stirring, solid APS was added for a $50 \mathrm{mM}$ final concentration. After $2 \mathrm{~h}$, the $\mathrm{pH}$ of the solution was 2.5. PABA was then purified by alkaline precipitation with $\mathrm{KOH}$ and redispersion in pure water (5 $\left.\mathrm{mg} \mathrm{mL} \mathrm{m}^{-1}\right)$.

The purified ZIF-8 nanocrystal methanolic colloids and PABA solutions were mixed in different proportions to obtain an increasingly higher PABA loading. The PABA content is expressed as weight percentage in the prepared sample, computed using the concentration of PABA solution and ZIF-8 colloidal suspensions (see Supporting Information for further details on characterization via X-ray diffraction (XRD), vibrational spectroscopy, and electronic microscopy).

Five powdered MOF samples were studied: pure MIL96(Al), pure ZIF-8, and ZIF-8 with three different loadings of $\mathrm{PABA}$. The samples were dried at $100{ }^{\circ} \mathrm{C}$ at a reduced pressure for $5 \mathrm{~h}$ and then placed in an isopiestic chamber for 5 days at ambient temperature. The chamber humidity was controlled using pure distilled water. ${ }^{44}$ Two separate NMR characterization procedures were carried out. One involved the loading of $4 \mathrm{~mm}$ MAS rotors, which were used to quantify the amount of adsorbed water by the acquisition of NMR spectra of ${ }^{1} \mathrm{H}$ at $300 \mathrm{MHz}$. A $4 \mathrm{~mm}$ solenoid corresponding to a Bruker miniimaging probe was used with a Bruker AVANCE II console. Background signals were acquired with an empty sample holder and later subtracted from each spectrum. For diffusion measurements, $200 \mathrm{mg}$ portions were pressed under 5 tons to form a $7.8 \mathrm{~mm}$ diameter pellet. To recover the water that may have been lost during the pressing process, the pellets were replaced on the isopiestic chamber for additional 5 days. After this period, the samples were hermetically sealed in $8 \mathrm{~mm}$ NMR glass tubes. The most general form of measuring diffusion in NMR is by the pulsed field gradient technique, where magnetic fields with constant gradients are applied for a short period of time, typically between 0.5 and $5 \mathrm{~ms}$. During the application, transverse magnetization is encoded by diffusion; however, this process is overimposed with transverse relaxation times, $T_{2}^{*}$. In the particular case of strongly adsorbed water molecules, $T_{2}^{*}$ may be extremely short, hindering the use of standard equipment. Recently, the diffusion of water molecules within a MOF matrix was measured using a home-built apparatus, capable of providing very short and strong gradient pulses. ${ }^{38}$ Another strategy is to use single-sided, or ex situ, NMR. ${ }^{42}$ These devices present a very intense magnetic field gradient which is used both to select an arbitrary volume within the sample and to codify the NMR signal by the undergoing diffusion processes. As the magnetic gradients in these devices are very strong, short encoding times of the order of tens to hundreds of microseconds may be used. Single-sided 
NMR has been used, for instance, for the determination of the mobility of liquids embedded in MOFs. ${ }^{31,37}$

A PM5 NMR-MOUSE (Magritek Ltd.) was used for the diffusion experiments. The device has a vertical penetration depth of $5.3 \mathrm{~mm}$, a resonance frequency of $20.28 \mathrm{MHz}$ for ${ }^{1} \mathrm{H}$, and a static gradient of $23.5 \mathrm{~T} / \mathrm{m}$. A slice of $140 \mu \mathrm{m}$ was excited in the center of the sample, and the diffusion measurements were carried out using a stimulated echo sequence (STE) with Carr-Purcell-Meiboom-Gil (CPMG) detection to improve sensitivity. ${ }^{42,45}$ The normalized signal attenuation for STE in the presence of a constant magnetic field gradient for the system used in this work (see Supporting Information for details) is given by

$$
\ln \left(\frac{S}{S_{0}}\right)=-\gamma^{2} G_{0}{ }^{2} \tau_{1}^{2}\left(\tau_{2}+\frac{2}{3} \tau_{1}\right) D=-b D
$$

where $\gamma$ is the gyromagnetic ratio of the nucleus, $G_{0}$ is the gradient strength, $\tau_{1}$ is the phase-encoding period, and $\tau_{2}$ is the longitudinal magnetization storage period. By changing the value of the encoding period and analyzing the signal decay, the diffusion coefficient value $(D)$ can be determined. The socalled $b$-value contains all relevant experimental parameters. In this way, fitting a linear relation provides the diffusion coefficient. Whenever different pools of molecules with different diffusion rates are present, multiple diffusion coefficients will be present. In this study, all data were described with a bilinear fitting, rendering two distinct diffusion coefficients.

\section{RESULTS AND DISCUSSION}

The analysis of water interaction with samples was carried out using a configuration aimed to understand the processes occurring in the synthesized MOF and MOF-polymer composites. Figure 1 shows the structures and the proposed
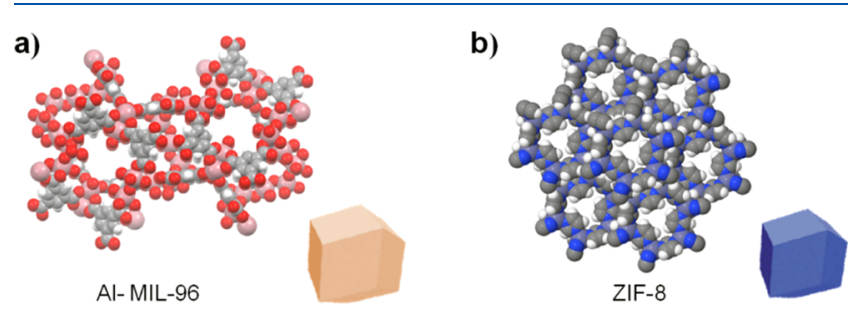

C)

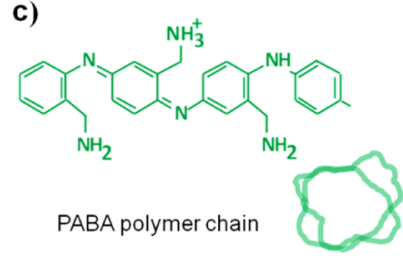

d)

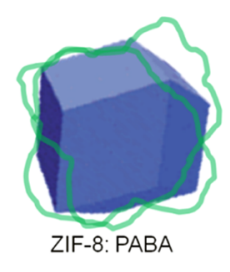

Figure 1. Structure and schematic representation of the MOFs AlMIL-96 (a) and ZIF-8 (b), polymer PABA (c), and ZIF/PABA composite (d).

schematic representations of materials involved in this study. Two different metal-organic frameworks were employed. On one side, Al-MIL-96 was chosen as the benchmark because of its hydrophilic nature, with the possibility to host water in the micropores (Figure 1a). In contrast, ZIF-8 presents $-\mathrm{CH}_{3}$ moieties at the pore windows which render a highly hydrophobic nature (Figure 1b). However, as the outer surface of ZIF-8 nanocrystals becomes partially covered with PABA (Figure 1c,d), the material becomes increasingly less hydrophobic.

The total amount of adsorbed water present in each system after exposure to saturation conditions can be quantified by the inspection of the high-field NMR spectra. Figure 2 shows a ${ }^{1} \mathrm{H}$

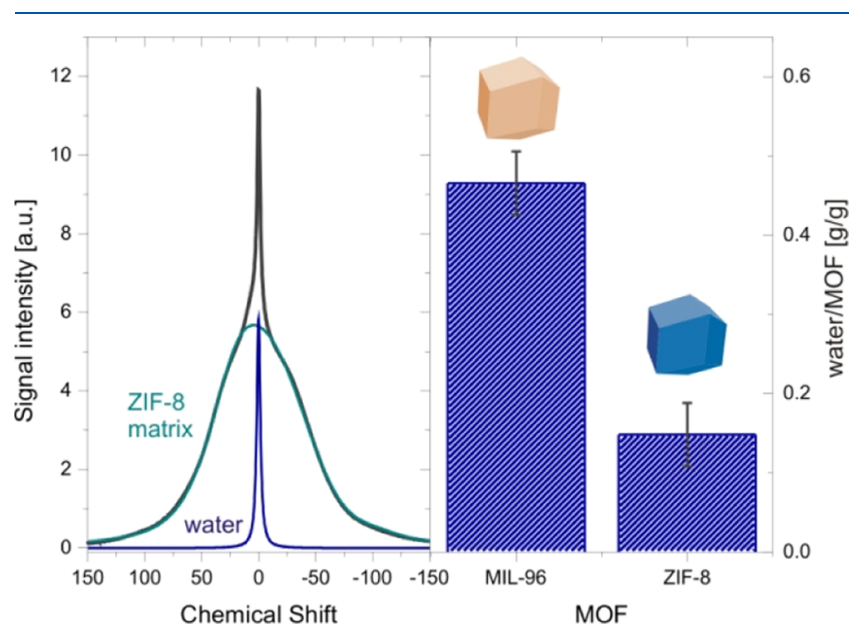

Figure 2. (left) High-field NMR spectrum of hydrated ZIF-8 (black line) with signal deconvolution showing the contribution of the porous matrix and the adsorbed water. (right) Water content in MIL96 and ZIF8.

spectrum for bare ZIF-8 after equilibration under such conditions. The total signal can be deconvoluted in two contributions. ${ }^{1} \mathrm{H}$ of the MOF matrix renders a broad signal, which is clearly separated from that of the narrower one assigned to adsorbed water. After the calibration of the spectrometer response with a reference water sample, the adsorbed amount of water per MOF mass can be obtained. MIL-96 is highly hydrophilic, whereas ZIF-8 microporosity is eminently hydrophobic, which can be inferred from the observed threefold water uptake increase when comparing the two samples. It is worth to mention that the water signal observed in the NMR spectra is proportional to the total amount of water, but for the materials considered, there are different environments for water molecules. In the MIL-96 framework, water most likely fills the available microporosity (or fundamental unit cages) with some amount of adsorbed water present in the spaces between the crystallite bundles. On the other hand, water cannot enter the highly hydrophobic microporous intracrystallite environments on ZIF- $8 ;{ }^{46,47}$ thus, the drastic reduction is observed.

NMR diffusion experiments portraying signal decay of water confined in MIL-96 are presented in Figure 3. The observed behavior confirms the existence of two different diffusional processes operating, that is, water molecules present in the sample are confined in different environments. Water molecules are adsorbed either within the MOF microporosity (intracrystallite space; $\mathrm{H}_{2} \mathrm{O}(3)$ in Figure 3 ) or in the intergrain space with meso-macroscopic dimensions, which allows an exchange between the adsorbed water and the vapor phase $\left(\mathrm{H}_{2} \mathrm{O}(1)\right.$ in Figure 3). Kärger developed a two-site exchange model for diffusion processes in similar systems. ${ }^{48}$ This model was recently applied to describe water diffusion kinetics within the MIL-100(Al) MOF material, which is a topological framework isomer of MIL-96, featuring the same building units linked through different spatial arrangements. ${ }^{38,49}$ The 

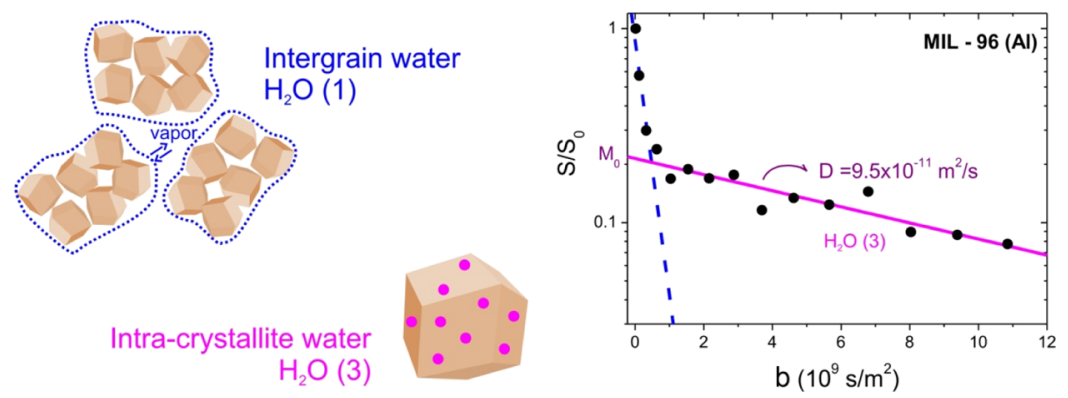

Figure 3. (left) Schematic representation of MIL-96 nanocrystals and different types of water confined in the micropores and in the intergrain space. (right) Experimental results displaying NMR signal decay of an STE in the presence of a constant magnetic field gradient. The fast decay (blue dashed line) is associated to water molecules in the intergrain (macro-/mesoporous) space, permanently exchanging between the adsorbed and vapor phases $\left(\mathrm{H}_{2} \mathrm{O}(1)\right)$. The slow decaying (magenta full line) component is associated with water in the intracrystallite (microporous) space $\left(\mathrm{H}_{2} \mathrm{O}(3)\right) . M_{0}$ is proportional to the number of molecules present in the microporosity.
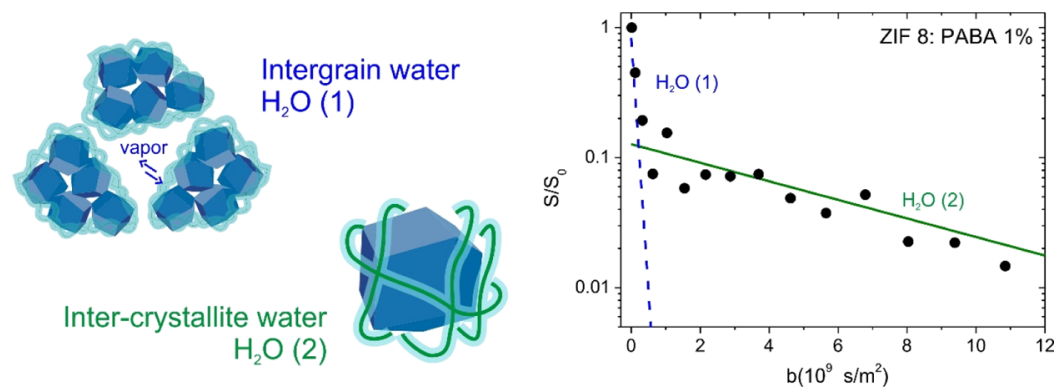

Figure 4. (left) Schematic representation of ZIF-8 nanocrystals modified with 1\% PABA and different behaviors of water present on the macromesoscopic regions and water confined on the modified crystallite surface. (right) Experimental results displaying NMR signal decay of an STE in the presence of a constant magnetic field gradient. The fast decay (blue dashed line) can be associated to the water molecules present in the macro-mesoscopic intergrain space $\left(\mathrm{H}_{2} \mathrm{O}(1)\right)$, featuring a rapid exchange with the vapor phase. The slow decaying component (green full line) can be related to the water molecules surface-confined to the PABA-modified ZIF-8 nanocrystals $\left(\mathrm{H}_{2} \mathrm{O}(2)\right)$.

initial fast decay observed in Figure 3 has been associated with the exchange of adsorbed water molecules with those in the vapor phase contained in the intergrain space. ${ }^{38}$ As the density in the liquid phase is several orders of magnitude larger than that of the gas phase, all of the detected NMR signals will arise from the liquid phase. However, as water molecules in these meso/macroporous spaces exchange between the adsorbed and gas phases repeatedly during the experimental time, molecules belonging to the gas phase acquire a large phase in the diffusion encoding period, compared to those in the adsorbed water phase. When this type of exchange is produced within the measurement time, the phase accumulation during the second $\tau_{2}$ time interval will be small, causing an erroneous determination of the diffusion coefficient, as reported by Splith et al. $^{38}$ In this situation, diffusion coefficients of 1 order of magnitude higher than those of freely diffusing water are obtained (with values around $8 \times 10^{-8} \mathrm{~m}^{2} / \mathrm{s}$ ), and therefore this part of the decay does not provide quantitative information on the confined water dynamics.

We will focus on the slow decay, which arises from the diffusion of water molecules present in the intracrystallite (microporous) space. A bilinear fitting allows to obtain two diffusion coefficients, and the extrapolation of the slow decay function renders the $M_{0}$ parameter, which is proportional to the amount of water present in the microporous environments during the experiment observational time (see Figure 3). ${ }^{38}$ In this way, the proportion of the NMR signal intensity corresponding to the MOF water loading at equilibrium conditions can be determined.
We now turn our attention to the experimental results obtained for pure ZIF-8 and PABA-modified ZIF-8 samples. For these samples, two diffusional regimes were also observed. However, as the ZIF-8 matrix is highly hydrophobic, the presence of water inside the crystal (similar to $\mathrm{H}_{2} \mathrm{O}(3)$ for MIL-96, as noted in Figure 2) can be ruled out. It can be assumed that the presence of increasingly higher amounts of polymer in the composite (which confer an increased hydrophilic character to the nanocrystal surface by the positioning of $-\mathrm{NH}_{2}$ moieties provided by PABA) leads to a new type of spatial confinement region where water molecules can be incorporated, as schematized in Figure 4. Polymer capping was demonstrated previously to be a suitable strategy for conferring hydrophilicity to an otherwise hydrophobic surface in a porous network. ${ }^{28}$ Moreover, similar confinement effect has been observed for porous polymer matrices with surface affinity toward water adsorption. ${ }^{50}$ Figure 4 shows schematically the proposed structures for ZIF-8/PABA composites allowing for a small fraction of water molecules to be adsorbed on top of the polymer-coated nanocrystals, which would continuously exchange with the vapor phase in contact with the intercrystallite space, leading to the observation of a fast decay in the STE experiment.

Because of the highly hydrophobic character of ZIF-8 micropores, the values obtained for the $M_{0}$ parameter (different from what was observed above for the MIL-96 material) should be proportional to the amount of water confined in the hydrophilic environment created by the PABA coating of the ZIF-8 nanocrystals (i.e., $\mathrm{H}_{2} \mathrm{O}(2)$ in a mesoscopic intercrystallite space). Figure 5 a shows that the 


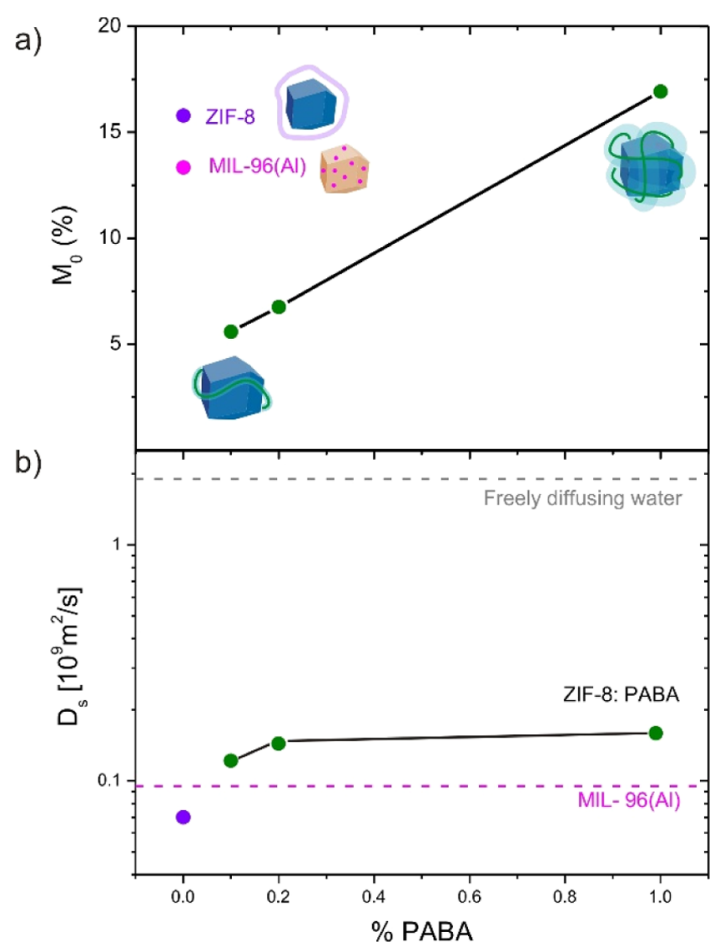

Figure 5. (a) Percentage of detected water $\left(M_{0}\right)$ in the environments defined by the presence of PABA and (b) diffusion coefficients of the slow-diffusing components as a function of weight percentage of PABA in the dry powder. Although both show an increase, the amount of water and diffusion coefficients can be observed as the polymer content is augmented; water amount increases linearly, whereas the diffusion coefficient value reaches (for relatively low PABA contents) a limit value.

$M_{0}$ value obtained for ZIF-8 is higher than the corresponding value determined for MIL-96. This implies that most of the water in the intercrystallite space in ZIF-8 is tightly adsorbed; thus, the fraction which is not able to perform molecular exchange with the gas phase is higher than the rest of the systems. This observation is confirmed in Figure $5 b$ by noting that the diffusion coefficient of this type of water in ZIF-8 has a very low value, even lower than that of water confined within MIL-96. Additionally, an increasing $M_{0}$ value is obtained as the PABA loading becomes higher, in line with the expected increment of the hydrophilic character. It is remarkable to note the fact that, even for the maximum PABA loading used in this work, the amount of signal corresponding to the water molecules in contact with the polymer-modified nanocrystal surface is lower than $20 \%$ of the total detected NMR signals. As PABA is incorporated in the structure, the diffusion coefficient of water increases with respect to MIL-96. This suggests that the slow-diffusing surface-confined water molecules on the polymer-MOF composite have a higher mobility than those in the hydrophilic MIL-96 benchmark MOF; even though the amount of incorporated water is lower, it is noted that for $0.1 \%$ PABA only $5 \%$ of the detected water is in the polymer-defined porous structure. Even when increasingly higher amounts of PABA on the composite allow the incorporation of more water, diffusion coefficients remain constant after reaching a limit value, that is, the mobility of these surface-confined water molecules is mainly affected by the physicochemical characteristics of the organicinorganic environment, and the amount of polymer added has a relatively moderate effect once a certain critical surface modification degree was achieved. This is consistent with the idea that the proportion of polymer-capped MOF nanocrystals increases with the PABA loading. Notably, the diffusion coefficients determined are 1 order of magnitude lower than that of freely diffusing water, denoting a strong mobility restriction.

\section{CONCLUSIONS}

Our results show that noncovalent modification procedures such as direct polymer capping can be used to tune the water hydrophobic/hydrophilic character of MOFs, and consequently, water mobility as well. By using hydrophilic Al-MIL$96 \mathrm{MOF}$ as the benchmark material, we have demonstrated that water affinity of ZIF-8 (an eminently hydrophobic MOF) can be increased by PABA inclusion. The NMR data reveal that the hydrophilicity of the composite materials must be understood not only in terms of the amount of adsorbed water but also in terms of the adsorbed water mobility. In view of multiple MOF film applications, and in particular, having in mind the recent findings pointing toward the use of ZIF-8/CP composite electrodes for enhancing the electrochemical ORR performance, $^{25,26}$ the gained insight contributes to the rationale of using such versatile novel functional nanoarchitectures. This opens the possibility of creating a composite material which combines features of ZIF-8 and PABA: high surface area MOF nanocrystals capable of gas adsorption, and both hydrophilicity (allowing migration of charged species present in aqueous solutions) and electrocatalytic properties of CPs modifying an otherwise insulator material such as ZIF-8 MOF.

\section{ASSOCIATED CONTENT}

\section{Supporting Information}

The Supporting Information is available free of charge on the ACS Publications website at DOI: 10.1021/acs.jpcc.9b06824.

Characterization (Fourier transform infrared spectroscopy, transmission electron microscopy, scanning electron microscopy, dynamic light scattering, and XRD) and synthesis of the materials used and a comprehensive explanation of the pulse sequence (STE-CPMG) used for the NMR diffusion measurements (PDF)

\section{AUTHOR INFORMATION}

\section{Corresponding Authors}

*E-mail: racosta@famaf.unc.edu.ar (R.H.A.).

*E-mail: mrafti@quimica.unlp.edu.ar (M.R.).

\section{ORCID}

Manuel I. Velasco: 0000-0003-1500-4672

Rodolfo H. Acosta: 0000-0003-3945-8273

Waldemar A. Marmisollé: 0000-0003-0031-5371

Omar Azzaroni: 0000-0002-5098-0612

Matías Rafti: 0000-0003-3393-358X

Notes

The authors declare no competing financial interest.

\section{ACKNOWLEDGMENTS}

M.I.V., R.H.A., W.A.M, M.R., and O.A. are CONICET fellows. The authors would like to acknowledge the financial support received from CONICET (PIP-11220130100619CO and 
$1111220130100746 \mathrm{CO})$, SeCyT-UNC, ANPCyT (PICT2014-0463, PICT-2015-0239, PICT-2016-1680, PICT-20102274, PICT-2014-1295 and PICT-2017-0957), and UNLP (PPID-X016).

\section{REFERENCES}

(1) McGillivray, L. R. Metal-Organic Frameworks; MacGillivray, L. R., Ed.; John Wiley \& Sons, Inc.: Hoboken, NJ, USA, 2010.

(2) Zhou, H.-C.; Long, J. R.; Yaghi, O. M. Introduction to MetalOrganic Frameworks. Chem. Rev. 2012, 112, 673-674.

(3) Furukawa, H.; Cordova, K. E.; O’Keeffe, M.; Yaghi, O. M. The Chemistry and Applications of Metal-Organic Frameworks. Science 2013, 341, 1230444.

(4) Stock, N.; Biswas, S. Synthesis of Metal-Organic Frameworks (MOFs): Routes to Various MOF Topologies, Morphologies, and Composites. Chem. Rev. 2012, 112, 933-969.

(5) Falcaro, P.; Hill, A. J.; Nairn, K. M.; Jasieniak, J.; Mardel, J. I.; Bastow, T. J.; Mayo, S. C.; Gimona, M.; Gomez, D.; Whitfield, H. J.; et al. A New Method to Position and Functionalize Metal-Organic Framework Crystals. Nat. Commun. 2011, 2, 237.

(6) Bétard, A.; Fischer, R. a. Metal-Organic Framework Thin Films: From Fundamentals to Applications. Chem. Rev. 2012, 112, 10551083.

(7) Cook, T. R.; Zheng, Y.-R.; Stang, P. J. Metal-Organic Frameworks and Self-Assembled Supramolecular Coordination Complexes: Comparing and Contrasting the Design, Synthesis, and Functionality of Metal-Organic Materials. Chem. Rev. 2013, 113, 734-777.

(8) Li, X.; Zhang, H.; Wang, P.; Hou, J.; Lu, J.; Easton, C. D.; Zhang, X.; Hill, M. R.; Thornton, A. W.; Liu, J. Z.; et al. Fast and Selective Fluoride Ion Conduction in Sub-1-Nanometer Metal-Organic Framework Channels. Nat. Commun. 2019, 10, 2490.

(9) Fracaroli, A. M.; Siman, P.; Nagib, D. A.; Suzuki, M.; Furukawa, H.; Toste, F. D.; Yaghi, O. M. Seven Post-Synthetic Covalent Reactions in Tandem Leading to Enzyme-like Complexity within Metal-Organic Framework Crystals. J. Am. Chem. Soc. 2016, 138, $8352-8355$.

(10) Chang, T.-H.; Kung, C.-W.; Chen, H.-W.; Huang, T.-Y.; Kao, S.-Y.; Lu, H.-C.; Lee, M.-H.; Boopathi, K. M.; Chu, C.-W.; Ho, K.-C. Planar Heterojunction Perovskite Solar Cells Incorporating MetalOrganic Framework Nanocrystals. Adv. Mater. 2015, 27, 7229-7235.

(11) Liu, J.; Zhou, W.; Liu, J.; Howard, I.; Kilibarda, G.; Schlabach, S.; Coupry, D.; Addicoat, M.; Yoneda, S.; Tsutsui, Y.; et al. Photoinduced Charge-Carrier Generation in Epitaxial MOF Thin Films: High Efficiency as a Result of an Indirect Electronic Band Gap? Angew. Chem., Int. Ed. 2015, 54, 7441-7445.

(12) Wu, D.; Guo, Z.; Yin, X.; Pang, Q.; Tu, B.; Zhang, L.; Wang, Y.G.; Li, Q. Metal-Organic Frameworks as Cathode Materials for Li-O 2 Batteries. Adv. Mater. 2014, 26, 3258-3262.

(13) Fracaroli, A. M.; Furukawa, H.; Suzuki, M.; Dodd, M.; Okajima, S.; Gándara, F.; Reimer, J. A.; Yaghi, O. M. Metal-Organic Frameworks with Precisely Designed Interior for Carbon Dioxide Capture in the Presence of Water. J. Am. Chem. Soc. 2014, 136, 88638866.

(14) Xiang, S.; He, Y.; Zhang, Z.; Wu, H.; Zhou, W.; Krishna, R.; Chen, B. Microporous Metal-Organic Framework with Potential for Carbon Dioxide Capture at Ambient Conditions. Nat. Commun. 2012, 3, 954-959.

(15) Burtch, N. C.; Jasuja, H.; Walton, K. S. Water Stability and Adsorption in Metal-Organic Frameworks. Chem. Rev. 2014, 114, 10575-10612.

(16) Kizzie, A. C.; Wong-Foy, A. G.; Matzger, A. J. Effect of Humidity on the Performance of Microporous Coordination Polymers as Adsorbents for CO2 Capture. Langmuir 2011, 27, 6368-6373.

(17) McDonald, K. A.; Feldblyum, J. I.; Koh, K.; Wong-Foy, A. G.; Matzger, A. J. Polymer@MOF@MOF: “Grafting from” Atom
Transfer Radical Polymerization for the Synthesis of Hybrid Porous Solids. Chem. Commun. 2015, 51, 11994-11996.

(18) Zhang, W.; Hu, Y.; Ge, J.; Jiang, H.-L.; Yu, S.-H. A Facile and General Coating Approach to Moisture/Water-Resistant MetalOrganic Frameworks with Intact Porosity. J. Am. Chem. Soc. 2014, 136, 16978-16981.

(19) Rafti, M.; Allegretto, J. A.; Segovia, G. M.; Tuninetti, J. S.; Giussi, J. M.; Bindini, E.; Azzaroni, O. Metal-Organic Frameworks Meet Polymer Brushes: Enhanced Crystalline Film Growth Induced by Macromolecular Primers. Mater. Chem. Front. 2017, 1, 2256-2260.

(20) Jeong, U.; Dogan, N. A.; Garai, M.; Nguyen, T. S.; Stoddart, J. F.; Yavuz, C. T. Inversion of Dispersion: Colloidal Stability of Calixarene Modified Metal-Organic Framework Nanoparticles in Non-Polar Media. J. Am. Chem. Soc. 2019, 141, 12182-12186.

(21) Sappia, L. D.; Piccinini, E.; Marmisollé, W.; Santilli, N.; Maza, E.; Moya, S.; Battaglini, F.; Madrid, R. E.; Azzaroni, O. Integration of Biorecognition Elements on PEDOT Platforms through Supramolecular Interactions. Adv. Mater. Interfaces 2017, 4, 1700502.

(22) Fenoy, G. E.; Giussi, J. M.; von Bilderling, C.; Maza, E. M.; Pietrasanta, L. I.; Knoll, W.; Marmisollé, W. A.; Azzaroni, O. Reversible Modulation of the Redox Activity in Conducting Polymer Nanofilms Induced by Hydrophobic Collapse of a Surface-Grafted Polyelectrolyte. J. Colloid Interface Sci. 2018, 518, 92-101.

(23) Wang, B.; Côté, A. P.; Furukawa, H.; O’Keeffe, M.; Yaghi, O. M. Colossal Cages in Zeolitic Imidazolate Frameworks as Selective Carbon Dioxide Reservoirs. Nature 2008, 453, 207-211.

(24) Park, K. S.; Ni, Z.; Cote, A. P.; Choi, J. Y.; Huang, R.; UribeRomo, F. J.; Chae, H. K.; O’Keeffe, M.; Yaghi, O. M. Exceptional Chemical and Thermal Stability of Zeolitic Imidazolate Frameworks. Proc. Natl. Acad. Sci. U. S. A. 2006, 103, 10186-10191.

(25) Rafti, M.; Marmisollé, W. A.; Azzaroni, O. Metal-Organic Frameworks Help Conducting Polymers Optimize the Efficiency of the Oxygen Reduction Reaction in Neutral Solutions. Adv. Mater. Interfaces 2016, 3, 1600047.

(26) Fenoy, G. E.; Scotto, J.; Azcárate, J. C.; Rafti, M.; Marmisollé, W. A.; Azzaroni, O. Powering up the Oxygen Reduction Reaction through the Integration of O2-Adsorbing Metal-Organic Frameworks on Nanocomposite Electrodes. ACS Appl. Energy Mater. 2018, 1, 5428.

(27) Rafti, M.; Marmisollé, W. A.; Azzaroni, O. Metal-Organic Frameworks Help Conducting Polymers Optimize the Efficiency of the Oxygen Reduction Reaction in Neutral Solutions. Adv. Mater. Interfaces 2016, 3, 1600047.

(28) Allegretto, J. A.; Tuninetti, J. S.; Lorenzo, A.; Ceolín, M.; Azzaroni, O.; Rafti, M. Polyelectrolyte Capping As Straightforward Approach toward Manipulation of Diffusive Transport in MOF Films. Langmuir 2018, 34, 425-431.

(29) Kong, X.; Scott, E.; Ding, W.; Mason, J. A.; Long, J. R.; Reimer, J. A. CO 2 Dynamics in a Metal-Organic Framework with Open Metal Sites. J. Am. Chem. Soc. 2012, 134, 14341-14344.

(30) Stallmach, F.; Gröger, S.; Künzel, V.; Kärger, J.; Yaghi, O. M.; Hesse, M.; Müller, U. NMR Studies on the Diffusion of Hydrocarbons on the Metal-Organic Framework Material MOF-5. Angew. Chem., Int. Ed. 2006, 45, 2123-2126.

(31) Chen, J. J.; Kong, X.; Sumida, K.; Manumpil, M. A.; Long, J. R.; Reimer, J. A. Ex Situ NMR Relaxometry of Metal - Organic Frameworks for Rapid. Angew. Chem. 2013, 125, 12265.

(32) Kärger, J.; Ruthven, D. M.; Theodorou, D. N. Diffusion in Nanoporous Materials; Wiley, 2012.

(33) Price, W. S. NMR Studies of Translational Motion. Principles and Applications; Cambridge University Press, 2009.

(34) Titze, T.; Lauerer, A.; Heinke, L.; Chmelik, C.; Zimmermann, N. E. R.; Keil, F. J.; Ruthven, D. M.; Kärger, J. Transport in Nanoporous Materials Including MOFs: The Applicability of Fick's Laws. Angew. Chem., Int. Ed. 2015, 54, 14580-14583.

(35) Ardelean, I.; Mattea, C.; Farrher, G.; Wonorahardjo, S.; Kimmich, R. Nuclear Magnetic Resonance Study of the Vapor Phase Contribution to Diffusion in Nanoporous Glasses Partially Filled with Water and Cyclohexane. J. Chem. Phys. 2003, 119, 10358-10362. 
(36) Mueller, R.; Hariharan, V.; Zhang, C.; Lively, R.; Vasenkov, S. Relationship between Mixed and Pure Gas Self-Diffusion for Ethane and Ethene in ZIF-8/6FDA-DAM Mixed-Matrix Membrane by Pulsed Field Gradient NMR. J. Membr. Sci. 2016, 499, 12-19.

(37) Witherspoon, V. J.; Yu, L. M.; Jawahery, S.; Braun, E.; Moosavi, S. M.; Schnell, S. K.; Smit, B.; Reimer, J. A. Translational and Rotational Motion of C8 Aromatics Adsorbed in Isotropic Porous Media (MOF-5): NMR Studies and MD Simulations. J. Phys. Chem. C 2017, 121, 15456-15462.

(38) Splith, T.; Pantatosaki, E.; Kolokathis, P. D.; Fröhlich, D.; Zhang, K.; Füldner, G.; Chmelik, C.; Jiang, J.; Henninger, S. K.; Stallmach, F.; et al. Molecular Dynamics Phenomena of Water in the Metalorganic Framework MIL-100(Al), as Revealed by Pulsed Field Gradient NMR and Atomistic Simulation. J. Phys. Chem. C 2017, 121, 18065-18074.

(39) Splith, T.; Fröhlich, D.; Henninger, S. K.; Stallmach, F. Development and Application of an Exchange Model for Anisotropic Water Diffusion in the Microporous MOF Aluminum Fumarate. J. Magn. Reson. 2018, 291, 40-46.

(40) Loiseau, T.; Lecroq, L.; Volkringer, C.; Marrot, J.; Férey, G.; Haouas, M.; Taulelle, F.; Bourrelly, S.; Llewellyn, P. L.; Latroche, M. MIL-96, a Porous Aluminum Trimesate 3D Structure Constructed from a Hexagonal Network of 18-Membered Rings and M3-OxoCentered Trinuclear Units. J. Am. Chem. Soc. 2006, 128, 1022310230.

(41) Furukawa, H.; Gándara, F.; Zhang, Y.-B.; Jiang, J.; Queen, W. L.; Hudson, M. R.; Yaghi, O. M. Water Adsorption in Porous MetalOrganic Frameworks and Related Materials. J. Am. Chem. Soc. 2014, 136, 4369-4381.

(42) Casanova, F.; Perlo, J.; Blümich, B. Single-Sided NMR; SpringerVerlag: Berlin Heidelberg, 2011.

(43) Marmisollé, W. A.; Maza, E.; Moya, S.; Azzaroni, O. AmineAppended Polyaniline as a Water Dispersible Electroactive Polyelectrolyte and Its Integration into Functional Self-Assembled Multilayers. Electrochim. Acta 2016, 210, 435-444.

(44) Greenspan, L. Humidity Fixed Points of Binary Saturated Aqueous Solutions. J. Res. Natl. Bur. Stand., Sect. A 1977, 81, 89.

(45) Carr, H. Y.; Purcell, E. M. Effects of Diffusion on Free Precession in Nuclear Magnetic Resonance Experiments. Phys. Rev. 1954, 94, 630-638.

(46) Sann, E. E.; Pan, Y.; Gao, Z.; Zhan, S.; Xia, F. Highly Hydrophobic ZIF-8 Particles and Application for Oil-Water Separation. Sep. Purif. Technol. 2018, 206, 186-191.

(47) Calero, S.; Gómez-Álvarez, P. Underlying Adsorption Mechanisms of Water in Hydrophobic and Hydrophilic Zeolite Imidazolate Frameworks: ZIF-71 and ZIF-90. J. Phys. Chem. C 2015, 119, 23774-23780.

(48) Kärger, J. Study of Fast Tracer Desorption in Molecular Sieve Crystals. AIChE J. 1982, 28, 417-423.

(49) Qiu, M.; Guan, Q.; Li, W. Controllable Assembly of Al-MIL100 via an Inducing Occupied Effect and Its Selective Adsorption Activity. Cryst. Growth Des. 2016, 16, 3639-3646.

(50) Silletta, E. V.; Velasco, M. I.; Gomez, C. G.; Strumia, M. C.; Stapf, S.; Mattea, C.; Monti, G. A.; Acosta, R. H. Enhanced Surface Interaction of Water Confined in Hierarchical Porous Polymers Induced by Hydrogen Bonding. Langmuir 2016, 32, 7427-7434. 